\title{
Anal canal pressure profile: a reappraisal as determined by rapid pullthrough technique
}

\author{
S M MCHUGH AND N E DIAMANT \\ From the Dept of Medicine, University of Toronto and Toronto Western Hospital, Toronto, Ontario, Canada
}

SUMmary Previous station pullthrough techniques of anal canal pressure measurement have not truly represented the differences among individuals and between sexes in the length and pressure profile of the anal canal. Furthermore, the techniques commonly used in anal canal pressure determination have not been adequately compared or standardised. Therefore, a mechanised rapid pullthrough technique using an 8-lumen, 4-quadrant, continuously perfused catheter was evaluated and used to define the anal canal pressure profile. For measurement of the resting anal canal pressures, this technique was compared with the more usual station pullthrough technique in 12 subjects. There were no differences in resting pressure recorded by either technique with infusion rates ranging from 0.5 to $1.2 \mathrm{cc} / \mathrm{min} /$ orifice and over a range of catheter withdrawal rates of () 19 to $(0.48 \mathrm{~cm} / \mathrm{sec}$. Twenty two subjects ( $12 \mathrm{men} / 10$ women $)$ had their anal canal pressure profiles assessed using the rapid pullthrough technique. Anal canal length representing the mean of four axes differed between the sexes $(\mathrm{p}<0.005)$ and the difference was largely accounted for by a decrease in length of the anterior axis in women. A standardisation technique was used to account for between subject variation and for a more accurate between sex comparison of the pressure profiles. Significant differences in radial symmetry were found between the sexes. In the anterior axis the pressure was higher distally in women, while in the anterior and lateral axes the pressure was higher proximally in men. Anteriorly in women the highest pressures were exerted over a much shorter canal length than in men. We conclude that the usual station pullthrough technique can give reliable resting pressure measurements in the anal canal. The rapid pullthrough technique described herein, however, used a flexible catheter system which follows the normal anal canal and rectal contours, allows a more appropriate assessment of the anal sphincter profile, and provides findings that are consistent with the known local anatomy.

A multitude of factors influence the measurement of the resting anal canal pressures. These include the technique used and the radial and longitudinal asymmetry along the anal canal. While biological variation in anal canal pressures is recognised, its contribution to sampling of anal canal pressures and the reproducibility of these measurements has not been well documented. Further, there is considerable variation in resting pressures and sphincter lengths among individuals of both sexes.

Pressures within the anal canal have been measured by water filled perfused catheter systems

Address for correspondence: Dr S M Mcllugh, Room 7-(014 ECW. Toronto Western Hospital. 399 Bathurst Street. Toronto. Ontario, Canada, M5T $2 \$ 8$. Received for publication 31 March 1987. using either a rapid pulthrough (RPT)' or more commonly, a station $(\mathrm{SPT})^{23}$ technique. No standarised technique is available, however. There are a number of potential disadvantages of the SPT techniques for certain aspects of measurement. In particular, it uses fixed points starting within the rectum, with intrarectal pressure as the zero $\mathrm{mmHg}$ reference point. If the sphincter is long, then the stations assessed will differ in comparison to those of a short anal canal. Also, it is technically difficult to manually do station pullthroughs accurately in many subjects because of buttock size and shape.

Therefore, we set out to compare the anal canal pressure profiles in men and women using: (a) a flexible perfused catheter which would permit four 
quadrant pressure measurement; (b) a mechanised rapid pullthrough (RPT) technique to more accurately determine the anal canal length and pressure profile in each of the four axes of the anal canal; and (c) a standardisation technique to take into account the between subject differences in either absolute pressures recorded or the length of the anal canal. The first part of this study assessed the RPT by (a) comparison with the SPT technique; and (b) determination of the effect of various perfusion rates and speeds of catheter withdrawal on the pressures recorded. A portion of this study has appeared in abstract form. ${ }^{+}$

\section{Methods}

\section{VOI.UNTEERS}

Twelve healthy volunteers underwent two separate anosphincteric manometric studies to assess the RPT technique in recording sessions lasting two to three hours. A further 10 subjects underwent a single recording session lasting about one hour using only the RPT technique. Rapid pullthrough data from these two groups of subjects totalling 22 individuals were then analysed to determine the anal canal profiles.

The Human Experimentation Committee of the University of Toronto approved the procedures used in this protocol in September, 1982.

Of the 22 subjects there were 10 women, mean age $27 \cdot 1 \pm 6 \cdot 2$ years and 12 men, mean age $26 \cdot 8 \pm 6 \cdot 3$ years. All subjects had a normal bowel habit, were free of systemic disease and had no previous anorectal complaints or surgery. None of the women had previously been pregnant.

An eight lumen polyvinyl catheter assembly (od $6 \mathrm{~mm}$ ) was constructed with four side openings arranged longitudinally $0.5 \mathrm{~cm}$ apart and transecting four orifices arranged radially. The latter were $15 \mathrm{~cm}$ from the catheter tip (Fig. 1). The catheter orifices were sufficiently proximal that withdrawal of the catheter for anal canal pressure determination did not result in the entire removal of the catheter. Repeat measurements were therefore possible by simple advancement of the catheter to reposition the orifices within the rectum. A $5 \mathrm{~cm}$ long latex balloon was fixed to the distal catheter to assess reflex

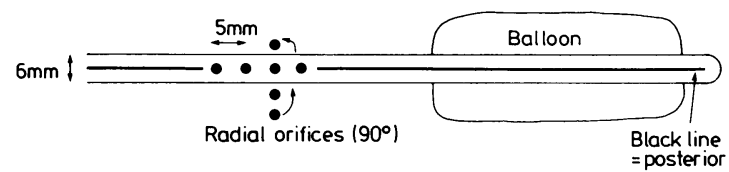

Fig. 1 Catheter assembly. Eight lumen polyvinyl perfused catheter (od $6 \mathrm{~mm})$. The balloon, used to assess anal sphincter reflexes, was not used in this study. sphincter responses to intrarectal balloon distension. The data derived from the reflex studies are not reported herein. The catheter was continuously perfused with water through a pneumohydraulic pump (Mui Scientific, Mississauga, Ontario) at rates of $0.5,0.7$, and $1.2 \mathrm{cc} / \mathrm{min} /$ orifice in the first set of experiments and at a rate of $0.7 \mathrm{cc} / \mathrm{min} /$ orifice in the second set of experiments. An eight channel direct writing recorder (Beckman 612) displayed pressures recorded via transducers (Beckman Type 4-327-C). The system recorded a pressure rise of greater than $200 \mathrm{mmHg} / \mathrm{sec}$.

The studies were carried out without bowel preparation. The subjects were studied in the left lateral position with hips flexed at 90 degrees. The recording catheter was inserted after a digital rectal examination, and five to 10 minutes allowed for basal conditions to re-establish. Rapid pullthrough measurements were done by a motorised withdrawal system clamped to the catheter and positioned so that the axis of traction followed the longitudinal axis of the anal canal. Rapid pullthroughs were carried out at constant rates of catheter withdrawal using a variable speed motor at speeds of $(0 \cdot 19,0.33,(0 \cdot 48$ $\mathrm{cm} / \mathrm{sec}$. These rates were accurately and consistently reproduced. Station pullthrough measurements were carried out by motorised withdrawal and stopping of the catheter at $0.5 \mathrm{~cm}$ stations. Peak pressures were recorded after maintaining catheter position for 4-5 seconds or until a stable baseline was recorded at each station. A black line marking the full length of the catheter was used to ensure the maintenance of the longitundinally spaced orifices in the posterior axis through the multiple pullthroughs.

The potential contribution of phasic activity to the resting sphincter pressure was assessed by a stationary recording lasting a minimum of five minutes with the catheter positioned such that the longitudinally arranged orifices traversed the anal canal. The amplitude and frequency of manometric phasic activity was determined.

In the first series of experiments the RPT and SPT were compared in 12 subjects under the following conditions: (a) differing infusion rates - that is, $0 \cdot 5$, 0.7 , and $1.2 \mathrm{cc} / \mathrm{min} /$ orifice; and (b) RPT speeds of $0 \cdot 19,0 \cdot 33$, and $(0 \cdot 48 \mathrm{~cm} / \mathrm{sec}$. In half of the subjects, six pullthroughs were performed for each variable in randomised order. In the remaining subjects, the RPTs were performed in consecutive sets of six for each variable. Approximately 250 pullthroughs were performed for each of the subjects in two recording sessions on separate days.

The highest resting pressure of each pullthrough profile was scored by a single observer using intrarectal pressure as the zero $\mathrm{mmHg}$ baseline. The start of the high pressure zone was defined as a rise over 


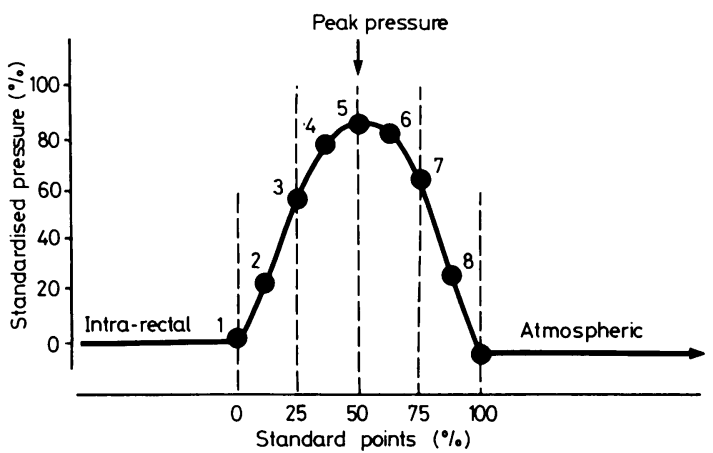

Fig. 2 Standardisation technique. Each RPT pressure profile was divided into four equal length segments with points 1 to 9 representing fixed percentages of the length of the anal canal high pressure zone. The pressures at each point were determined using intra-rectal pressure as the zero $\mathrm{mm} \mathrm{Hg}$ reference, and expressed as a percentage of the maximal peak pressure recorded in multiple $R P T$ (Standardised pressure).

rectal pressure of greater than $4 \mathrm{mmHg}$. The resting pressure was initially expressed as the mean of six pullthroughs (averaging the four axes) and was determined for each experimental variable. Each subject's resting pressure was ultimately calculated as the mean of six RPT done at the beginning and end of each recording session at an infusion rate of 0.7 $\mathrm{cc} / \mathrm{min} /$ orifice and a withdrawal rate of $0.33 \mathrm{~cm} / \mathrm{sec}$. Knowing both paper and RPT speed, the length of the sphincter could be measured directly.

The pressure profiles of all 22 subjects ( 12 men, 10 women) were standardised for the right, anterior, left, and posterior axes using data derived for six RPT at a withdrawal speed of $0.33 \mathrm{~cm} / \mathrm{sec}$ and infusion rate of $0.7 \mathrm{cc} / \mathrm{min} /$ orifice. The standardisation technique consisted of dividing each pressure profile into four equal segments and the pressures were determined at the ends and the midpoints of each segment yielding nine points of measurement. For each subject, the pressures recorded at each of the nine points for the six RPT were averaged (four quadrants separately) and then converted to a percentage of the highest resting pressure recorded to permit comparison between subjects (Fig. 2). Profiles were constructed for both men and women and were analysed statistically comparing the nine points on each axis between the two sexes.

The pressure profiles between men and women appeared to be different in shape particularly in the anterior axis. Therefore, for each sex and for each axis, the length of the anal canal high pressure zone exerting at least $60,65,70$, and $75 \%$ of the peak resting pressure was calculated. The pressures at the standard points along the right and left axes were averaged to represent a single lateral axis.
STATISTICAL ANALYSIS

All measurements cited are given as the mean \pm SD. The results for the 12 subjects assessed to compare the techniques were analysed statistically by analysis of variance on grouped means for each variable. The anal canal pressure profiles were analysed using Student's $t$ tests to compare each of the nine points on each axis between the two sexes. (Statistical Analysis Systems Package, University of Toronto.)

\section{Results}

TECHNIQUE VALIDATION

The resting pressures recorded by either of the pullthrough techniques at any of the withdrawal speeds were not different using infusion rates between 0.5 and 1.2 cc/orifice ( $p>0.05$ ) (Fig. 3). In addition, the pressures recorded using the rapid pullthrough technique were not different at catheter withdrawal rates ranging from 0.19 to $0.48 \mathrm{~cm} / \mathrm{sec}$. Finally, there were no significant differences in resting pressures recorded by either the rapid or station pullthrough techniques (Fig. 3).
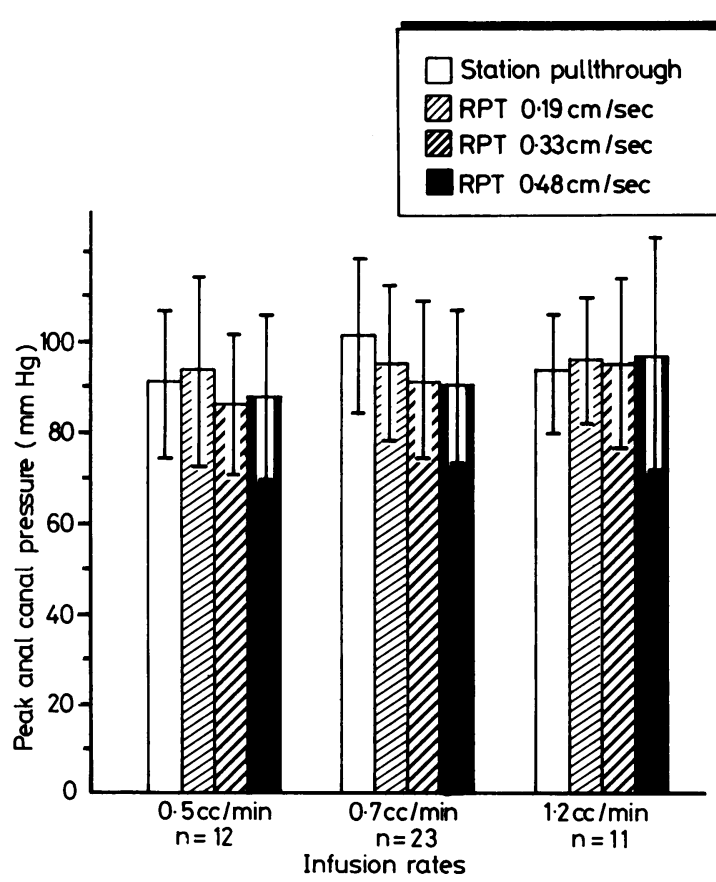

Fig. 3 Effect of infusion rate and pullthrough technique on peak anal canal pressures. No statistically significant differences were found between either the speed of RPT withdrawal or the infusion rates utilised. (RPT-rapid pullthrough). At $0.7 \mathrm{cc} / \mathrm{min}$, the study was repeated on each of two separate recording days in 11 of the 12 subjects resulting in $n=23$. 


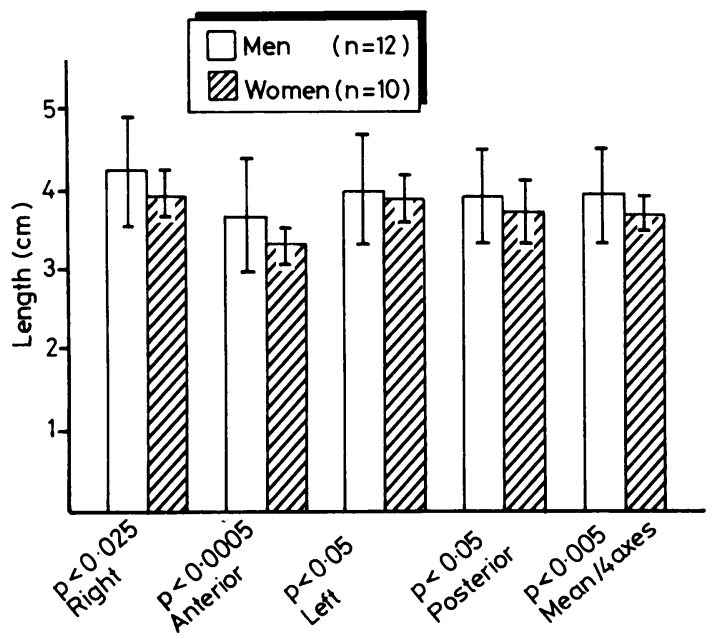

Fig. 4 Anal canal length. The length in women was significantly shorter in the anterior and lateral axes. The anal canal length calculated as the mean of the four axes was significantly shorter in women, largely because of the contribution of the anterior axis.

\section{RESTING PRESSURE DETERMINATION}

The resting pressures for men with a mean age of $26 \cdot 8 \pm 6 \cdot 3$ years was $94 \cdot 4 \pm 18 \cdot 3 \mathrm{mmHg}$ and was not significantly different from the pressures in women with a mean age of $27 \cdot 1 \pm 6 \cdot 2$ years which was $103 \cdot 9 \pm 23 \mathrm{mmHg}(\mathrm{p}>0 \cdot() 5)$.
In 11 of the subjects studied on two separate days, the resting pressures (day $1-99.8 \pm 16.7 \mathrm{mmHg}$; day $2-100 \cdot 3 \pm 28 \cdot 4 \mathrm{mmHg}$ ) were not significantly different $(p>0 \cdot 05)$. The within individual variation on successive days was less than $12 \%$ for all 11 subjects.

\section{ANAL CANAL PRESSURE PROFIIE}

Sphincter length

The length of the anal canal (mean of four axes) was significantly shorter $(\mathrm{p}<0) \cdot(0) 5)$ in women $(3 \cdot 7 \pm 0.2$ $\mathrm{cm})$ compared with men $(4 \cdot() \pm() \cdot 6 \mathrm{~cm})$ (Fig. 4). In most of the females ( 9 of 10) the anterior axis was the shortest $(3 \cdot 3 \pm() \cdot 2 \mathrm{~cm})$ and significantly different $(\mathrm{p}<0.0005)$ from the anterior axis in men $(3.7 \pm 0.7$ $\mathrm{cm})$. In men there were no significant differences among the axis lengths. In women most of the difference in the overall sphincter length was attributable to a shorter anterior axis.

\section{Anal canal pressure profile}

There was no consistent radial asymmetry of the pressure profile in either men or women or between the two sexes. In women, the highest pressure in the canal was recorded in the anterior axis approximately $8 \mathrm{~mm}$ from the anal verge and this pressure was significantly higher than those recorded in other axes at this point $(\mathrm{p}<0 \cdot(0005)$. In men, the pressure in the lateral axes tended to be slightly higher along the canal (Fig. 5).

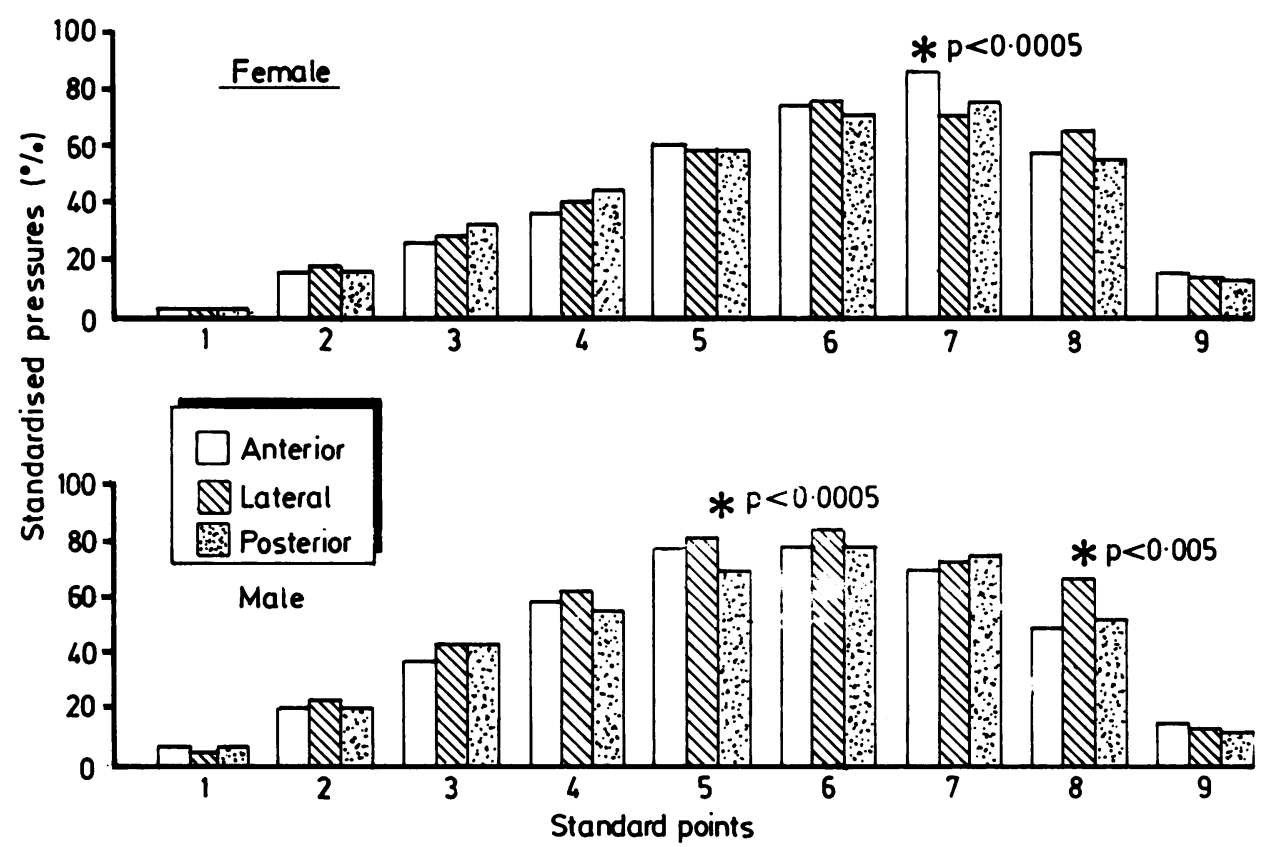

Fig. 5 Radial profile of the anal canal: Within sex differences. The anterior and posterior axes are compared with the combined left and right axes (expressed as the lateral axis), at nine standard points along the anal canal. 


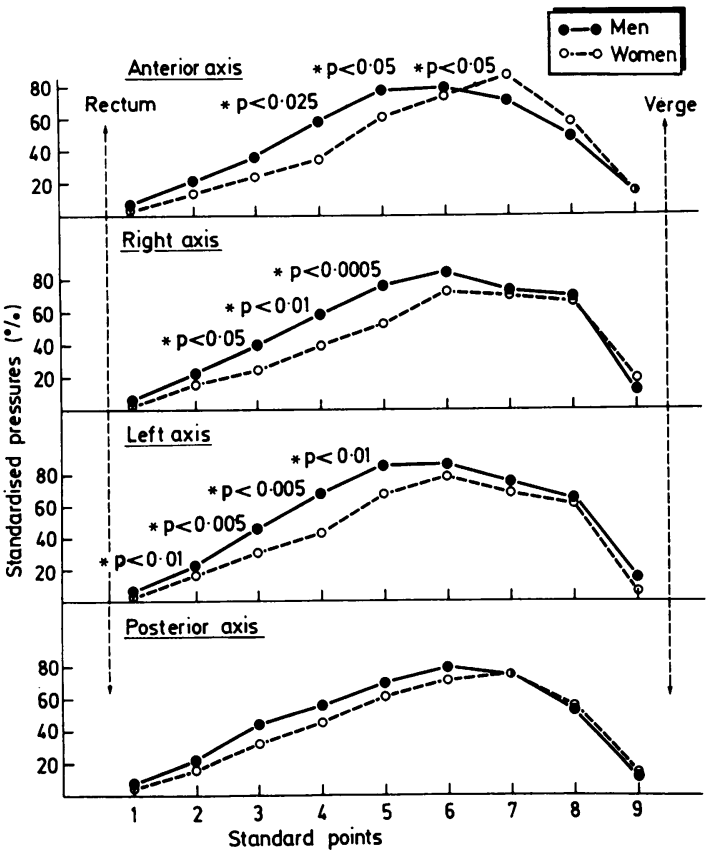

Fig. 6 Resting anal canal pressure profile between sex differences. Differences are noted only in the anterior and lateral axes with higher pressures noted in men in the more proximal part of the anal canal in all three axes. Pressures were significantly higher in the distal anterior axis in women.

When comparing women with men there were consistent differences in the pressure profile in the long axis of the canal. This was most evident anteriorly and laterally. Of particular note, in women the peak pressures were seen more distally, while in men the pressures were higher in the more proximal canal (Fig. 6). The anal canal length in each axis that exerts a pressure which is at least $60,65,70$, or $75 \%$ of the average resting pressure is outlined in the Table. This length is shown diagramatically in relation to the true length of the anal canal and the accepted anatomy of this region (Fig. 7). For a percentage of peak pressure that is $65 \%$ or greater, this length in

Table Length $(\mathrm{cm})$ of anal canal exerting different percentages of the peak resting pressure

\begin{tabular}{|c|c|c|c|c|c|c|c|c|}
\hline \multirow[b]{2}{*}{ Axis } & \multicolumn{2}{|c|}{$>60 \%$} & \multicolumn{2}{|c|}{$>6.5 \%$} & \multicolumn{2}{|c|}{$>70 \%$} & \multicolumn{2}{|c|}{$>75 \%$} \\
\hline & $F$ & $M$ & $F$ & $M$ & $F$ & $M$ & $F$ & $M$ \\
\hline Anterior & 0.82 & 0.92 & 0.41 & 0.92 & 0.41 & 0.92 & $*$ & 0.96 \\
\hline Posterior & 0.92 & 0.98 & 0.46 & 0.98 & 0.46 & 0.49 & $*$ & 0.49 \\
\hline Latcral & 1.47 & $2 \cdot(1) 4$ & 0.98 & 1.53 & $(0.49$ & $1 \cdot() 2$ & $*$ & 0.46 \\
\hline
\end{tabular}

$\mathrm{M}=$ male; $\mathrm{F}=$ female: ${ }^{*}=$ only one point exerting this pressure. no length determinable
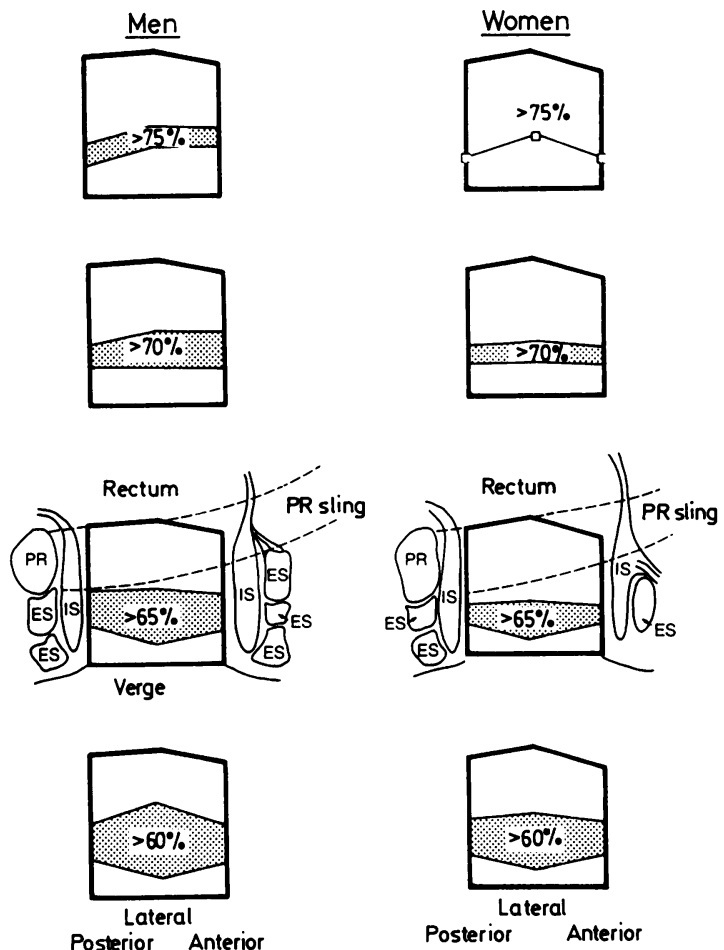

$\%$ Of anal canal HPZ $\square$ Anal canal

Fig. 7 Position of highest pressures recorded in each axis of the anal canal. The length $(\mathrm{cm})$ and position of anal canal exerting at least $60,65,70$, and $75 \%$ of peak pressure (shaded areas) is shown in relation to the true mean anal canal lengths in the anterior, posterior and lateral axes. On one set of diagrams, the differences in the local anatomy are illustrated schematically as described by Oh and Kark (1972).

$P R=$ puborectalis, $I S=$ internal sphincter,$E S=$ external sphincter.

the anterior axis of the anal canal of men $(0.92 \mathrm{~cm})$ is at least two times the length in the same axis in women $(0.41 \mathrm{~cm})$.

\section{PHASIC ACTIVITY}

Approximately one quarter of subjects (six of 22) demonstrated manometric 'ultraslow' phasic contractile activity with a frequency range of approximately one to two per minute and a mean amplitude of $63 \pm 33 \mathrm{mmHg}$. In the majority of the remaining subjects (12 of 16), there was inconsistent noncyclical pressure variation in the sphincter which was of considerably less amplitude than the ultraslow activity $(35 \pm 11 \mathrm{mmHg})$. All subjects demonstrated more rapid manometric phasic contractile activity that varied throughout the sphincter both with respect to its frequency and amplitude. The fre- 
quency of this type of activity was $10 \pm 2$ per minute in the proximal canal and $18 \pm 3$ per minute in the distal canal. The amplitude of this phasic activity tended to be higher in the proximal canal $(18 \pm 8 \mathrm{mmHg})$ than in the distal canal $(11 \pm 5 \mathrm{mmHg})$. There was considerably more variation in the appearance of the pressure profile and the resting pressures recorded in those individuals in whom the manometric ultraslow phasic activity was most prominent (Fig. 8 for example). Respiratory rates were routinely assessed. None of the variation in phasic anal pressures corresponded with respiratory rate changes.

\section{Discussion}

The anal canal is a potential space delineated by the surrounding musculature and defined manometrically as a zone of increased pressure. Continence to solid or liquid faecal material within the rectum is in part related to this resting high pressure zone which represents the contribution of both the smooth and striated muscle sphincters.

The manometric phasic activity observed in the resting anal canal was similar to previous reports. ${ }^{25}$ Low amplitude slow phasic activity was apparent in all subjects but ultraslow activity was noted in only one-quarter of individuals. No attempt was made to exclude those subjects with prominent ultraslow activity despite the considerable change in the anal canal pressure profile throughout the course of each cycle. Measurement of anal canal pressures using a mechanised rapid pullthrough technique has previously been reported " but not compared with the more usual station pullthrough techniques. We have shown that slow steady withdrawal of the recording catheter through the anal canal at a rate in the range of 0.19 to $0.48 \mathrm{~cm} / \mathrm{sec}$ does not affect the resting pressures recorded and, in particular, does not apparently lead to an increase in the voluntary sphincter contribution to the resting pressures as has been often assumed. ${ }^{*}$ The resting pressures recorded by the RPT technique did not differ from those recorded by the SPT technique. Therefore, either method is satisfactory for the determination of resting anal canal pressures. Distinctive pressure profile differences, however, were apparent between men and women when multiple four quadrant assessments were done by the rapid pullthrough method after standardising the anal canal pressure profiles.

Our study has shown that the infusion rates commonly used in the performance of oesophageal manometry yield pressure recordings that are unchanged with higher infusion rates. Therefore, infusion rates of $0.5-1.2 \mathrm{cc} / \mathrm{min} /$ orifice can be used without fear of a pressure change caused by the infusion rate.

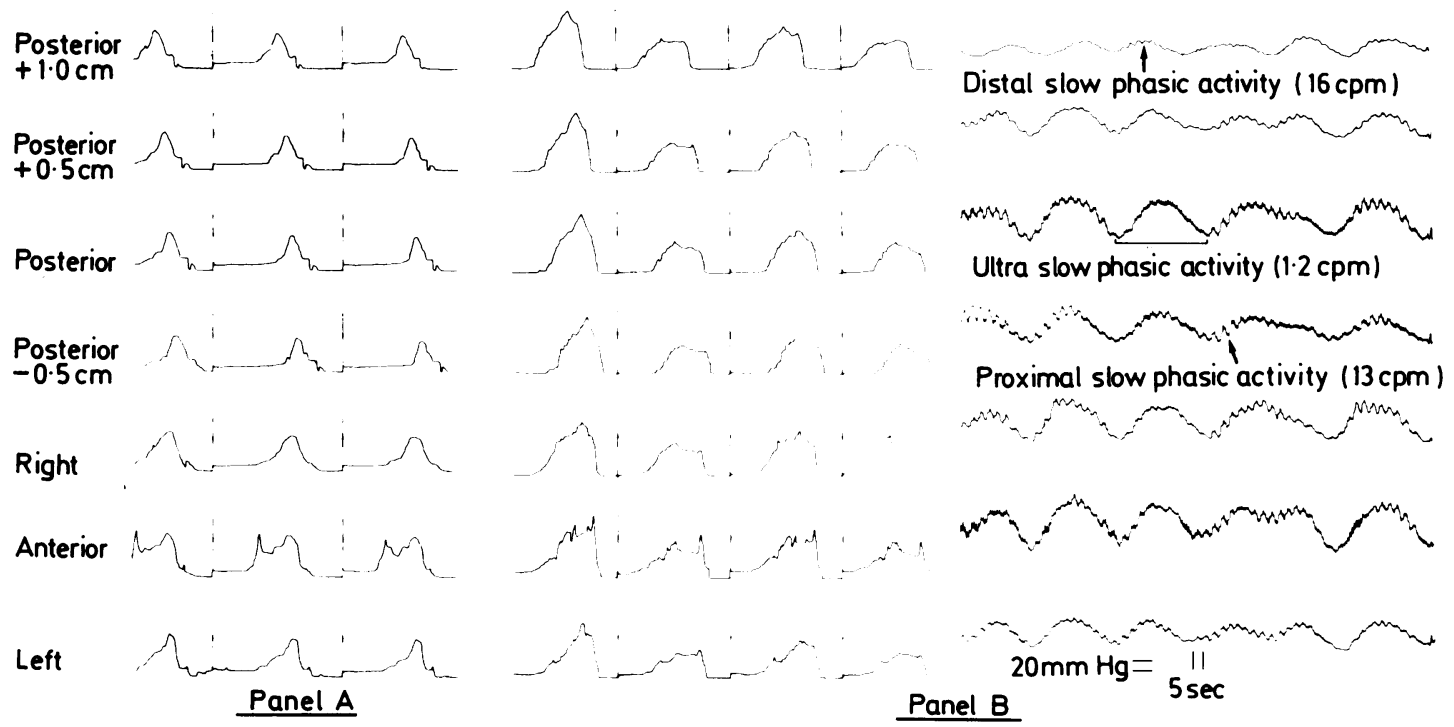

Fig. 8 Mechanised anal canal RPT effect of manometric phasic activity. Panel A shows multiple RPT in an individual without prominent ultraslow phasic contractile activity. The two sets of tracings in panel $B$ are from an individual with ultraslow activity with an amplitude up to $100 \mathrm{mmHg}$, the left set represents multiple RPT, the right set are derived with the catheter stationary in the anal canal. Tracings 1 to 4 are longitudinally arranged at $0.5 \mathrm{~cm}$, while tracings 5 to 7 are radially arranged in the anterior, right and left axes respectively, intersecting with 3. The reproductibility of any pressure profile relates to the phasic variation found. In the absence of ultraslow activity the pressure profiles are much more reproducible in appearance as shown in panel $A$; with ultraslow activity, the pressure profiles are more variable as shown in panel $B$. 
As speed of catheter withdrawal was constant and known, the length of the high pressure zone of the anal canal could be accurately measured. Our findings are consistent with previous reports ${ }^{34}$ in that the anal canal is shorter in women than in men but only when considering the average of the four axes. Our results show that the length of the posterior axis of the anal canal is the same for both men and women but the anterior axis is significantly shorter in women. The lateral axes appear intermediate between the anterior-posterior dimensions in both sexes. As the outer margins of the anal canal are all at the same level, these differences in axis length must reflect the shape of the proximal border of the anal canal with the axes beginning at different points.

In addition to differences in axis length, the pressures in the anal canal are known to vary along the longitudinal axis ${ }^{2}$ "' with the highest pressures variously reported to occur at $1 \mathrm{~cm}^{11}$ and $2 \mathrm{~cm}^{1112}$ from the anal verge, or halfway along the anal canal. ${ }^{3} \mathrm{We}$ also noted pressure differences along the anal canal and this longitudinal pressure profile differed between men and women.

Because pressures recorded along the anal canal and anal canal length vary, the pressure profiles were standardised to allow comparison of individuals and between sexes. Pressures at each point along each axis were considered as a percentage of the highest pressure recorded in that axis and distance as a percentage of the anal canal length. Using these standardisation techniques only minor differences in the radial symmetry were noted within each sex. When the anal pressures along the canal were compared between sexes, however, the pressures in the lateral and anterior axes (but not the posterior) differed. In the anterior axis the pressure was higher distally in women, while in both the anterior and lateral axes the pressure was higher more proximally in men.

Taylor et al using a rigid probe and a station pullthrough technique to record anal canal profiles, reported that pressures were reduced at the anterior proximal station $(1 \mathrm{~cm}$ from rectum) and the posterior distal station ( $1 \mathrm{~cm}$ from the anal verge). ${ }^{3}$ As Taylor et al point out, however, the positioning of their rigid pressure probe to follow the sacral concavity of the rectum could affect the pressures recorded. This would be likely to occur if their pressure probe rotated around the puborectalis, when the resistance of the anal wall upon the probe would be expected to produce lower pressures in the distal posterior station and the proximal anterior station. We have not shown the same radial asymmetry described by Taylor et al and suggest this reflects our use of a flexible catheter system which more readily follows the normal contours of the anorectum. In our view, the proximal decreased pressure anteriorly is due to the shorter anterior axis of the anal canal.

Not only did we show the anterior anal canal to be shorter in women but also the length of sphincter in this axis exerting the highest pressure was reduced in comparison to men. The length of the anterior anal canal exerting at least $65 \%$ of the resting pressure comprised less than half of the same length in men. This observation is consistent with the anatomical descriptions of Oh and Kark who performed careful dissection of the external sphincter and perineum in men and women. ${ }^{13}$ They found that the sphincter structures were considerably reduced in women in the anterior position compared with men. Also, the puborectalis muscle does not surround the anterior anal canal and, its absence may contribute to reduced resting pressures in this location. ${ }^{8}$ The very short total length, the length of highest pressure zone and its distal location in women anteriorly present significant risk to major sphincter disruption with either a generous episiotomy or a perineal tear during childbirth into the rectum.

This study shows that there are distinct differences between men and women in their anal canal pressure profiles when carefully assessed using recording instruments that do not deform the anal canal, a recording technique that gives a continuous profile of the radial and axial pressures, and a standardisation technique which allows for intersubject variation. These between sex differences are compatible with previously described anatomical descriptions of the anal sphincter. The more complete anal canal pressure profiles can be readily determined by a rapid pullthrough technique. Between sex differences in these profiles must be taken into account with future detailed studies of such events as reflex responses. For simple estimation of the resting anal canal pressure, however, multiple measurements by either the station pullthrough technique or rapid pullthrough technique yield similar values, and these values are not different over a considerable range of infusion rates and speed of catheter withdrawal.

Supported through the Elsie Watt Fund and MRC Grant 3353.

\section{References}

1 Kielmann J, Bonnesen T. Anal profilometry, a method of investigating the physiology of the anal canal and the pelvic floor. Dan Med Bull 1985; 35: 61-3.

2 Gutierrez JG, Oliai A, Chey WM. Manometric profile of the internal anal sphincter in man. [Abstract]. Gastroenterology 1975; 68: 907. 
3 Taylor BM, Beart, RW Jr, Phillips SF. Longitudinal and radial variations of pressure in the human anal sphincter. Gastroenterology 1984; 86: 693-7.

4 McHugh SM, Diamant NE. Anal canal pressure profile by rapid pullthrough technique. [Abstract]. Dig Dis Sci 1984; 29: 550 .

5 Hancock BD. Measurement of anal pressure and motility. Gut 1976; 17: 645-51.

6 Abrams PH. Perfusion urethral profilometry. Urol Clin NA 1979, 6: 103-9.

7 Bubrick MP, Godec CJ, Cass AS. Functional evaluation of the rectal ampulla with ampullometrogram. $J R$ Soc Med 1980; 73: 234-7.

8 Read NW, Bannister JJ. Anorectal manometry: Techniques in health and anorectal disease. In: Henry MM,
Swash M, eds. Coloproctology and the pelvic floor. London: Butterworths, 1985.

9 Nivatvongs MD, Stern HS. Fryd DS. The length of the anal canal. Dis Col Rectum 1981; 24: 60)-1.

10 Hill JR, Kelly ML. Shlegel JF, Code CF. Pressure profile of the rectum and anus of healthy persons. Dis Col Rectum 1960); 3: 203-9.

11 Collins CD, Duthie HL, Shelley T, Whittaker GE. Force in the anal canal and anal incontinence. Gut 1967; 8: $354-60$.

12 Duthie HL, Watts, JM. Contribution of the external anal sphincter to the pressure zone in the anal canal. Gut $1965 ; 6$ : 64-8.

13 Oh C, Kark AE. Anatomy of the external anal sphincter. Br J Surg 1972; 59: 717-23. 